\title{
Context effects in judging taste intensity: A comparison of variable line and category rating methods
}

\author{
J. A. STILLMAN \\ University of Auckland, Auckland, New Zealand
}

\begin{abstract}
The effect of stimulus range and stimulus spacing was examined when subjects registered the perceived intensity of sweet liquids using either a matching procedure (Experiment 1) or category rating (Experiment 2). The matching procedure is conceptually similar to absolute magnitude estimation, whereby subjects match their impression of number size to their impression of the subjective magnitude of a stimulus. In Experiment 1, subjects matched their impression of the stimulus to their impression of the magnitude of the length of a continuously variable line under their control. In Experiment 2, subjects rated perceived sweetness on a vertical 13-point scale with five equally spaced verbal labels. In both experiments, three sets of four sucrose concentrations were employed. In two of these sets, a set of weaker solutions and a set of stronger solutions, concentrations were separated by $0.25 \mathrm{log}$ units. In a third set, which spanned the range of concentrations used in the other two sets $(0.87$ to $27.36 \%$ w/v), solutions were separated by $0.5 \mathrm{log}$ units. An examination of both individual and group data showed the matching procedure to be less susceptible to a range bias than the rating procedure. In particular, a single intensity function accommodated data from individual ranges better when the matching procedure was used than when the rating procedure was used. No effect of stimulus spacing was evident in the data from either procedure.
\end{abstract}

The efficacy of scaling procedures is the subject of continuing debate within psychophysics. Much of the debate focuses upon the unreliability of subjects' estimates of sensory magnitude, attributable in large measure to an adaptive tendency to appraise sensory quantities within the context of their environment (Birnbaum, 1982; Laming, 1984; Lawless \& Malone, 1986; Lockhead, 1992; Parducci, 1974). Nevertheless, the development of acceptable methods for the registration of the intensity of sensory qualities is of considerable practical importance, nowhere more so than within the food industry. In the last decade, several pieces of research have suggested that a technique known as "absolute magnitude estimation" (AME) is less biased by stimulus context than magnitude estimations in which a standard stimulus is employed. AME requires subjects to match their subjective impression of the size of a number to their subjective impression of the intensity of a stimulus without reference to a modulus. I share with Zwislocki (1991) the view that the demands of AME are simpler than those of other scaling methods because, in contrast to magnitude estimation using a modulus where subjects must assign numbers in proportion to the ratio of their sensations, AME places

The author is grateful to R. J. Irwin for helpful comments on a draft of this manuscript and to Lawrence E. Marks and two anonymous reviewers for useful and constructive comments on an earlier version. The author is now at Massey University Albany, Private Bag 102904 , North Shore MSC, Auckland, New Zealand. upon subjects the simple requirement of judging whether two sensory magnitudes are equal. ${ }^{1}$ Recognizing the equality of two sensory magnitudes is fundamentally different from estimating sensory ratios or distances, and ought to reduce that component of the judgmental variance attributable to inaccuracies in specifying the amount by which two unmatched quantities differ or the ratio in which they stand. For example, in the ordinary sense (in contrast to the exacting specifications of physics), it is a simple matter to judge whether a stick and a piece of string are the same length or whether the stick is longer or shorter than the string. However, if it is necessary to specify by what proportion or amount one exceeds the other, an additional judgment is called for. (According to a view first proposed by Marks, 1974, interval scaling methods such as category ratings provide estimates of sensory distances, while ratio-scaling methods provide estimates of sensory magnitude. Thus, although the two types of scale are nonlinearly related, both may produce valid results.) Furthermore, if sensory quantities need only be matched to each other, one might suppose that context will be relatively unimportant, because relationships between sensations are preserved in the face of contextual change.

Zwislocki and Goodman (1980), Gescheider (1988), Collins and Gescheider (1989), and Gescheider and Hughson (1991), concluded that AME reduced bias. By contrast, Foley, Cross, and O'Reilly (1990) found that absolute magnitude estimates of the areas of triangles were affected by the subrange of previously judged stimuli. Ward (1987) obtained relatively small context effects using 
absolute magnitude estimation, but contends that judgments generally tend to be relative rather than absolute.

In fact, despite the generally improved outcomes that have been achieved experimentally, the theoretical assumptions underlying AME do not entail that its outcomes are exempt from experimental biases (see the discussion between Mellers, 1983a, 1983b, and Zwislocki, 1983a). The assumption underlying AME is that subjects make judgments of sensation magnitude on an absolute rather than a ratio scale. Improvements are expected relative to the use of a standard stimulus with a defined modulus, because subjects are not compelled to use numbers the perceived sizes of which do not match the sensation magnitudes of the stimuli, thereby generating distorted psychophysical scales.

The assumption that a comparison between subjective impressions originating in different senses is feasible is strengthened by a growing body of research demonstrating the existence of cross-sensory interactions between perceived qualities of perceptual experience (Marks, 1975, 1978, 1987; Melara \& O'Brien, 1987; Mudd, 1963), and is consistent with R. Teghtsoonian's (1973) suggestion that sensation magnitudes share a common range, irrespective of the dynamic stimulus range particular to each sense. In this paper, comparisons between sensory magnitudes are assumed to be comparisons between like qualities, while comparisons between incommensurable qualities, for example between line-length and sweetness or between loudness and brightness, are assumed not to be possible.

Most studies using AME have been conducted in the sense of hearing. This study investigates stimulus context and stimulus-spacing biases in the sense of taste, using a sensory matching technique that is a variant of AME. The technique shares the concept of matching and the $\mathrm{ab}$ sence of a modulus with AME, but differs from it in that the sensory impression created by the stimulus is compared to that created by the length of a line. In this respect, the procedure is a member of the class of cross-modality matching techniques advocated by Stevens (1975). The technique improves on older line-length matching procedures (see, e.g., Collins \& Gescheider, 1989; Hellman \& Meiselman, 1990) inasmuch as the length of the line is continuously variable and under subject control. The use of line-length matching assumes that the perception of line length is veridical. This assumption is supported by a number of experiments demonstrating that magnitude estimation of line length is proportional to physical line length (Bogartz, 1979; M. Teghtsoonian, 1965; Verrillo, 1981; Zwislocki, 1983b; Zwislocki \& Goodman, 1980).

In this study, the magnitude of the subjective impression created by sweet liquids at various concentrations was matched to that produced by a line, without reference to judgments given previously. With this procedure, the advantages of AME are utilized in the absence of the cognitive effort required to generate numbers prior to the comparison of sensory magnitudes. Effectively, the sub- ject has only to report a match when the magnitude of the impression created by the physically present variable stimulus (the line) corresponds to that produced by the fixed stimulus (the sample of sweet liquid). The influence of stimulus context on line-length matches is compared to its influence on ratings obtained by a procedure used by others to scale sweetness (McBride, 1983a, 1983b, 1986, 1987a; Riskey, Parducci, \& Beauchamp, 1979). The aim of this study was to compare two methods for registering sensory magnitudes, with respect to well-documented biases in responding induced by context (see, e.g., Riskey et al., 1979, and Marks et al., 1988, for evidence of context effects in the sense of taste). It should, however, be acknowledged that judgmental changes might not be the only effect of stimulus context. Recently, Marks (1992, 1993; Rankin \& Marks, 1991) and Schneider and Parker (1990) have explored the important possibility that context effects are at least partly perceptual. Gescheider and Hughson (1991) acknowledged the possibility that a residual context effect in their study might be attributable to perceptual changes. In the present study, only the manner in which sensory magnitudes are registered differed, and the same set of stimulus contexts was used with each technique. Context effects arising at an earlier locus than the responding stage would therefore be identical, leaving differences in the magnitude of context effects to be attributed to a subsequent judgmental stage.

\section{EXPERIMENT 1}

This experiment examined the prevalence of two common biases (Poulton, 1979, 1987) in group and individual data obtained using the line-length matching technique. The first of these biases is the range bias, whereby in the extreme the subject utilizes a fixed range of responses irrespective of the stimulus range. The second bias is the stimulus spacing bias, ${ }^{2}$ whereby responses from various regions of the response range are used about equally often irrespective of how the stimuli are spaced, thus producing an increase in the slope of the response function with decreasing stimulus spacing.

\section{Method}

Subjects. The subjects were 20 nonsmoking volunteers, students at the University of Auckland, who had no known diseases of the mouth or oral cavity, and who had been free of viral infections for the 3 weeks prior to the collection of data.

Stimuli. The stimuli were samples of food-grade sucrose in distilled water made up at least $24 \mathrm{~h}$ prior to testing and refrigerated and used within 3 days of mixing. Samples were presented in individual 40-ml-capacity portion cups with lids, arranged in numbered locations on a tray. Each cup contained a 15-ml sample. Three concentration ranges were used: a weaker range, a stronger range, and an extended range spanning the other two ranges. Concentrations in the weaker and stronger ranges were spaced $0.25 \mathrm{log}$ units apart, and the strongest concentration in the weaker range served as the weakest concentration in the stronger range. Concentrations in the extended range were spaced $0.5 \mathrm{log}$ units apart. In all, a total of seven different concentrations were used, five of them on two occasions each, in the context of different ranges. The four concen- 
trations in each range were: weaker range, $0.87 \%, 1.54 \%, 2.74 \%$, and $4.87 \%$ (weight/volume); stronger range, $4.87 \%, 8.65 \%$, $15.39 \%$, and $27.36 \%(\mathrm{w} / \mathrm{v})$; and extended range, $0.87 \%, 2.74 \%$, $8.65 \%$, and $27.36 \%(w / v)$. Expressed as molar concentrations, these values ranged from approximately 0.025 to $0.85 \mathrm{M}$. If sucrose threshold is taken as $0.015 \mathrm{M}$, then, in terms of McBride's (1987b) "taste coefficient," the range of taste responses expected to these stimuli is 0.12 to 0.82 . This ratio is based on the Beidler taste equation, and varies between asymptotes $0=$ no taste, and $1=$ saturation.

Line-length generation. Each line was generated on a Macintosh computer. The computer screen was hidden from the subject, who saw the line projected, by means of a Sayett Datashow projection pad, onto a $110 \times 130 \mathrm{~cm}$ screen approximately $3 \mathrm{~m}$ distant. The line expanded horizontally beginning at the center of the screen as soon as the subject depressed the mouse button, and continued to grow in length so long as the button was depressed. Once the button was released, a subsequent press decreased the length of the line until the button was released again. Alternate button presses increased and decreased the length of the line until the subject considered that his or her impression of the magnitude of the sweetness of the solution was matched by his or her impression of the magnitude of the length of the line. At the conclusion of a trial, a small arrow, parked in a rectangle labeled "Go" at the bottom left of the screen, was moved to an identical rectangle labeled "FINISH" at the bottom right of the screen. The length of the line in pixels was then automatically converted to the actual projected length, accurate to the nearest $0.5 \mathrm{~cm}$, and stored in the computer alongside a record of the sucrose concentration sampled on that trial. The conversion was made relative to a calibration line used when the apparatus was set up.

Procedure. The subjects were given practice in the operation of the mouse button to generate a line, and the procedure was explained to them prior to the commencement of the experimental trials. The subjects were asked to imagine little and big lines, and little and big sensations of sweetness. They were told that their task was to produce a line, their impression of the magnitude of which matched their impression of the magnitude of the sweetness of the solution. They were also instructed to judge each sample on its own merits, without reference to previous judgments. Random orderings of the 12 samples in each session (3 repeats of each of 4 concentrations) were computer generated through the implementation of a permutation algorithm by Brysbaert (1991). A unique order was thus used

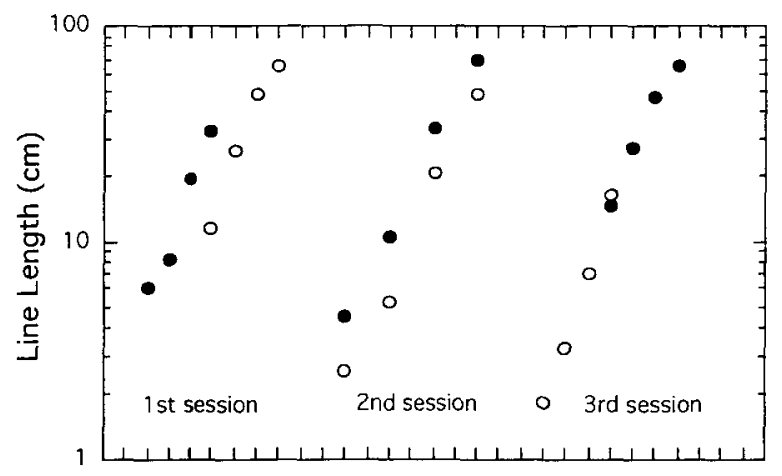

Relative concentration ( $1 \mathrm{div}=0.25 \log$ units)

Figure 1. Geometric means of line lengths used to represent sweetness as a function of the relative concentration of sucrose solutions in each of three sessions. Closed circles represent the group that received the set of weaker concentrations in the first session, and open circles represent the group that received the set of stronger concentrations in the first session.

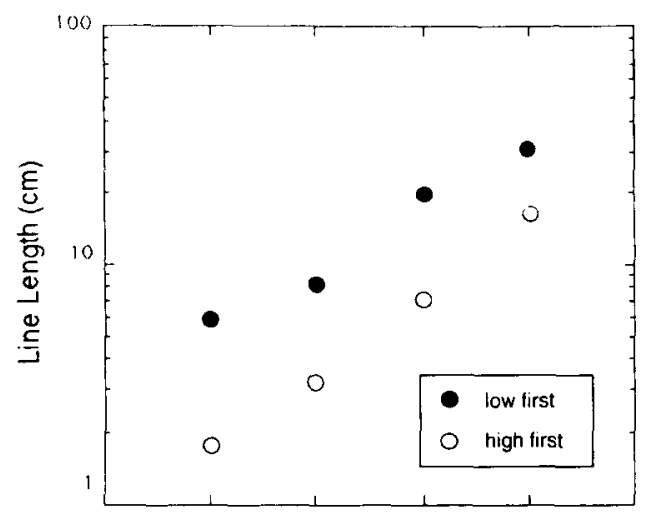

Relative concentration ( $1 \mathrm{div}=0.25 \log$ units)

Figure 2. Geometric means of line lengths used to represent the sweetness of the set of low sucrose concentrations as a function of the relative concentration of sucrose solutions. Symbols have the same meaning as in Figure 1.

by each subject in each of three sessions, one per week for 3 weeks. All subjects judged the extended range of concentrations in the second session. Half of the subjects judged the weaker range in the first session and the stronger range in the third session; the remainder of the subjects judged these two ranges in the reverse order. Solutions were presented at room temperature, approximately $21^{\circ} \mathrm{C}$, and the subjects were instructed to sample and expectorate each in accordance with the "sip and spit" technique commonly used in food science. Rinsing with distilled water was mandatory between trials.

\section{Results}

Group data. The average line lengths produced in response to the four concentrations presented during each session are displayed in Figure 1. Because the matching procedure is analogous to magnitude estimation, the data are geometric means and log values of line length are used in all the analyses (Zwislocki \& Goodman, 1980). The log-normal nature of the data was confirmed by univariate analysis. The closed circles in Figure 1 are data from the group that judged the weaker solutions in the first session, and the open circles are data from the group that judged the stronger solutions in the first session. Inspection of the figure shows that individuals who encountered the weaker concentrations first (closed circles) used somewhat longer lines to represent solutions in the weaker and extended concentration ranges than those who judged the stronger concentrations first. (Compare the closed circles for the first session in Figure 1 with the open circles for the third session, and compare the closed and open circles for the second session.) To facilitate comparison in the weaker range, the judgments of both groups are presented together in Figure 2.

In their first sessions, the two groups did not represent the single concentration common to the weaker and stronger ranges $(4.87 \% \mathrm{w} / \mathrm{v})$ by the same line. However, they did not use identical response ranges (extreme range bias) because, with this one exception, the lines used to repre- 
sent the stronger solutions were longer than those used for the weaker solutions. By the third session, the lines used by the two groups to represent the concentration common to the weaker and stronger sets were in excellent agreement. The trends evident in Figure 1 were confirmed by a general linear model analysis of variance, with the order of sessions as a between-groups factor and stimulus range and concentration as within-group factors. (The data of 1 subject who produced lines that were little affected by concentration level were omitted from the analysis and figures. When subsequently questioned, she stated that she had greatly disliked the solutions and had produced lines representing a "protest vote"). At the .05 level of significance, there was a main effect for group $[F(1,17)=5.11, p<.037]$ and for range $[F(2,34)=$ $56.86, p<.0001]$. These effects were modified by a significant group $\times$ range interaction $[F(2,34)=5.99, p<$ $.005]$, in the light of which the data in each range were analyzed individually. These individual analyses revealed a significant difference between the groups only with respect to the weaker concentration range $[F(1,17)=$ $8.17, p<.011]$ and not with respect to the extended or stronger ranges.

In summary, weaker concentrations were represented by longer lines if they were the first set encountered than if they were the third set encountered, whereas stronger concentrations were judged equivalently irrespective of the order in which the stimulus sets were encountered. By the third session, the data from the two groups appear to lie on a single function, showing no evidence of a range effect. Because equivalent judgments were given by both groups to the stronger concentrations, the disappearance of the range effect between Sessions 1 and 3 indicates a shift over the course of the experiment in the response range of the group that tasted the weaker concentrations first.

Individual data. Figure 3 shows the line-length adjustments of individual subjects. The group who judged the weaker solutions first are represented in the upper panel, and the group who judged the stronger solutions first are represented in the lower panel. In each panel, open squares are used for the extended range, open circles for the weaker range, and closed circles for the stronger range. Lines are drawn by eye through data in order to unambiguously identify individual subjects.

In the absence of a range effect, the closed circles should lie above the open circles except at the location of the common stimulus that served as the strongest concentration in the weaker range and the weakest concentration in the stronger range. For some individuals, the range effect is

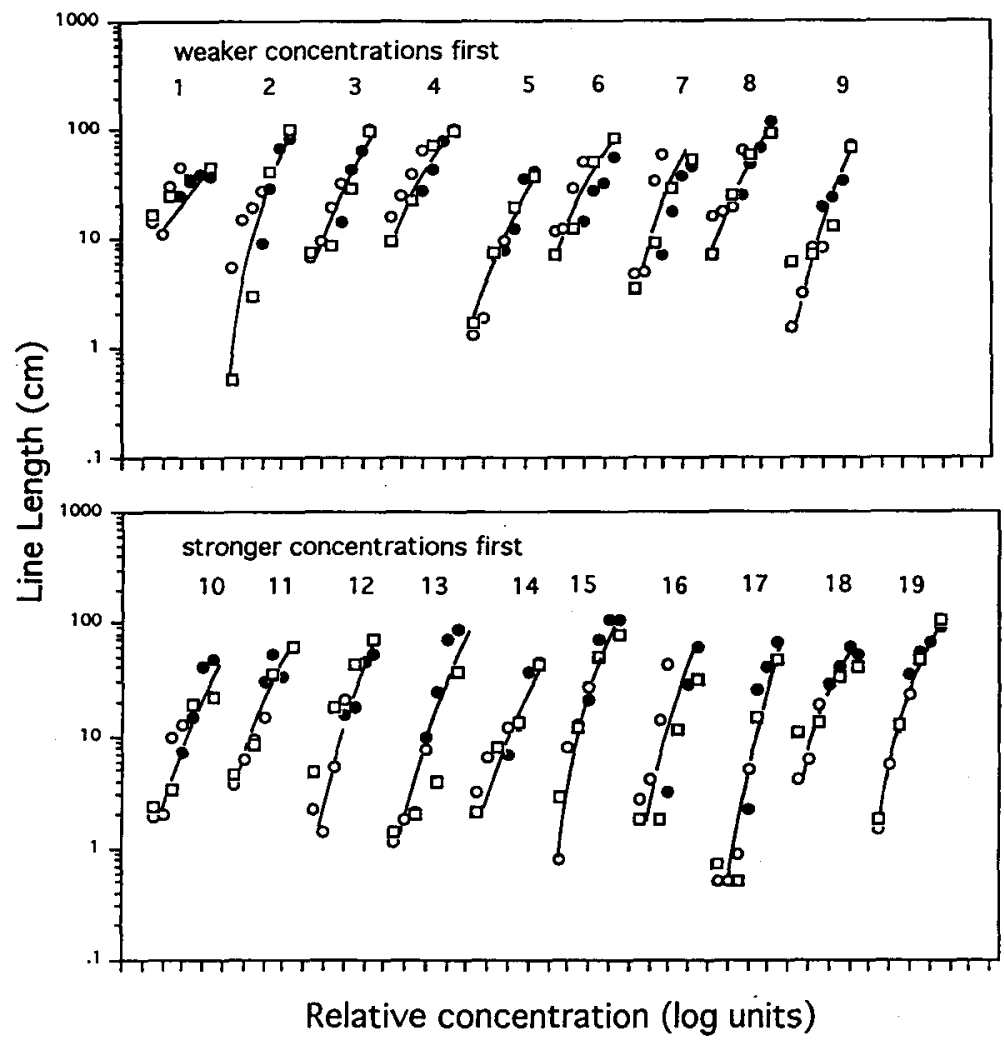

Figure 3. Line lengths used by individual subjects to represent sweetness, as a function of the relative concentration of sucrose. In each panel, the open circles represent the weaker range, the closed circles, the stronger range, and the open squares, the extended range. The two panels segregate the subjects by the order in which they judged the sets of weaker and stronger concentrations. 
slight; for others, it is more apparent. There is little evidence of a stimulus spacing bias in any of the data from Experiment 1.

\section{Discussion}

There was some effect of experiencing stimulus range with this procedure, in that subjects who encountered the weaker set of concentrations in their first block of trials matched these to longer lines than did subjects who encountered these concentrations in their third block of trials. There is the suggestion of an analogous effect in Figure 2 of Gescheider and Hughson (1991), who used AME to scale loudness, but in that study the effect was not statistically significant. In the present experiment, the weakest stimulus was close to the threshold for sucrose, and subjects who judged the weak solutions first may have feit reluctant to offer negligible lines, even when these actually represented a match to their impression of sweetness. This possibility is supported by the reduced difference between the line lengths used by this group to represent the two weakest solutions. That is, the inflation in line length is greatest for the weakest solution. However, judgments for the entire set of weak solutions are shifted toward larger values, and it may be that once the judgments for the weakest stimulus became inflated, other members of the set were affected because subjects hesitated to use similar lines to represent what were evidently stronger experiences.

There is no suggestion of a stimulus spacing bias in either the group or the individual data, in that the slope of the intensity functions is not less in the extended range than in the other two ranges comprising concentrations spaced half as far apart.

\section{EXPERIMENT 2}

A second experiment was undertaken, using the same sucrose concentrations as in Experiment 1, to determine whether the matching technique used in Experiment 1 was less prone to the influence of context than are category rating procedures.

\section{Method}

Subjects. The subjects were 18 nonsmoking volunteers, students at the University of Auckland, who had no known diseases of the mouth or oral cavity, and who had been free of viral infections for the 3 weeks prior to the collection of data. None had participated in Experiment 1.

Stimuli. The stimuli used in Experiment 2 were the same as those used in Experiment 1, and they were presented in the same three ranges.

Response scale. The scale comprised a vertical column partitioned by horizontal lines into 13 boxes (McBride, 1987a). On the left of the column were five equally spaced verbal descriptors going from extremely sweet, (at the top of the column) through very sweet, moderately sweet, slightly sweet to no sweetness at all (at the bottom of the column).

Procedure. As in Experiment 1, two groups of subjects were used, a group that judged the weaker concentrations first and a group that judged the stronger concentrations first. As before, the subjects attended once per week for 3 weeks. A unique permutation of the 12 solutions presented at each session was used for each subject.

\section{Results}

Group data. The ratings given to the various concentrations of sucrose in each of the three sessions are displayed in Figure 4. On analysis, the group $[F(1,16)=$ $6.71, p<.0197]$ and range $[F(2,32)=64.90, p<$ .0001 ] main effects were significant beyond the .05 level. These effects were modified by a significant group $\times$ range interaction $[F(2,32)=9.34, p<.0006]$. Further analysis revealed that the largest difference between the groups was in the ratings given to the weaker concentrations $[F(1,16)=16.90, p<.0008]$. In Experiment 1 , this was the only range for which a significant difference was found. In Experiment 2, the groups also differed significantly in their ratings of the extended range of concentrations $[F(1,16)=4.93, p<.0411]$, but not in their

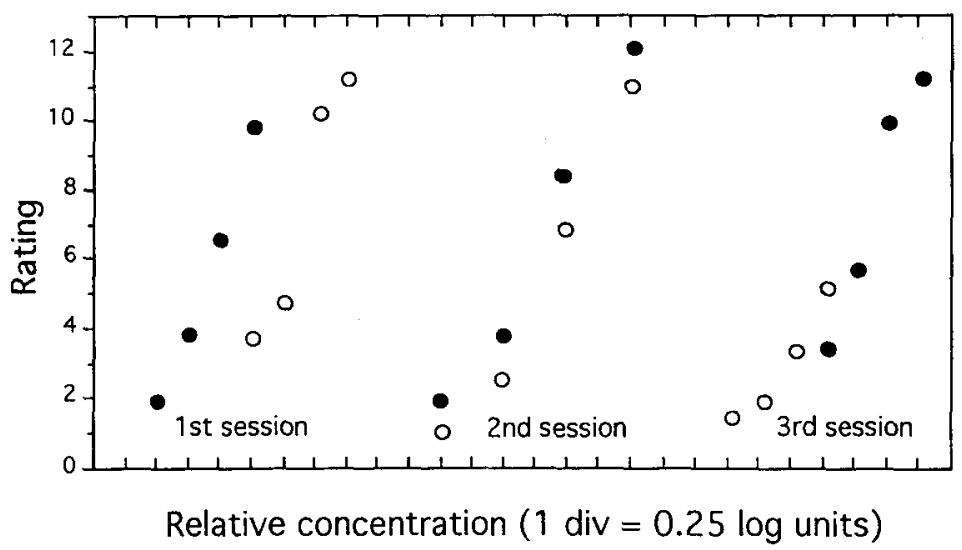

Figure 4. Arithmetic means of ratings of sweetness as a function of the relative concentration of sucrose solutions in each of three sessions. Closed circles represent the group that received the set of weaker concentrations in the first session, and open circles represent the group that received the set of stronger concentrations in the first session. 


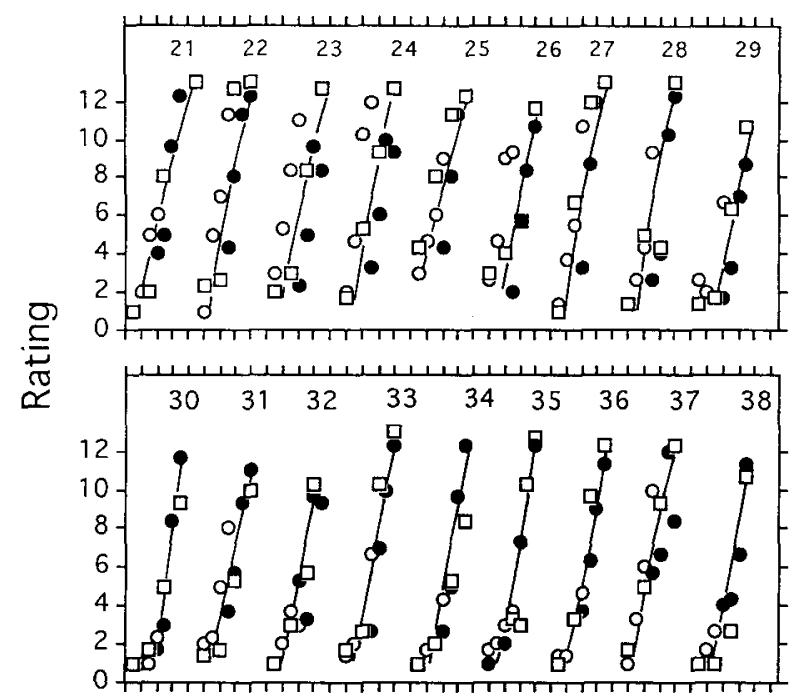

Relative concentration ( $1 \mathrm{div}=0.5 \log$ units)

Figure 5. Ratings of sweetness by individual subjects as a function of the relative concentration of sucrose. In each panel, the open circles represent the weaker range, the closed circles, the stronger range, and the open squares, the extended range. The two panels segregate the subjects by the order in which they judged the weaker and stronger concentrations.

ratings of the stronger concentrations $[F(1,16)=0.01$, $p<.9386]$.

Individual data. Individual ratings given to the various sucrose concentrations are shown in Figure 5, where the symbols have the same meaning as in Figure 3. There is a pronounced effect of stimulus context in the individual data. The range of responses given to weaker and stronger concentrations overlaps to a greater extent than might be predicted from the average data shown in Figure 4.

\section{Discussion}

The statistical analysis of the ratings from Experiment 2 , as well as the greater overlap in that experiment between ratings for the weaker and stronger ranges (compare Figures 1 and 2 with Figures 4 and 5), suggests a greater effect of stimulus range in Experiment 2 than in Experiment 1. Despite experience with the solutions in the first two sessions, the data from the two groups did not lie on congruent intensity functions at Session 3, as they did with the matching procedure. Since the intensity functions are not markedly shallower in the extended range than in the other ranges, there is, as before, little suggestion of a stimulus spacing bias.

\section{GENERAL DISCUSSION}

The purpose of these experiments was to compare the effect of stimulus context when subjects used either a continuously variable line or a category rating scale to represent sensory magnitude. The extent to which the judg- ments of sensory magnitude were independent of the range in which the stimuli occurred can be gauged from the extent to which judgments in the various concentration ranges were accommodated by a single function relating sensory magnitude to stimulus magnitude. To this end, the best-fitting functions appropriate to the two procedures were found, specifically a power function for the average data from Experiment 1 (Stevens's law) and a log function for the average data from Experiment 2 (Fechner's law; see McBride, 1983a). The parameters of the best-fitting functions were then compared with those fitted separately to the data in each range. The parameters were found by computer implementation of an algorithm that takes account of the variance of the individual points and finds the best-fitting function to the linearized data using chi-square minimization. Returned are the model
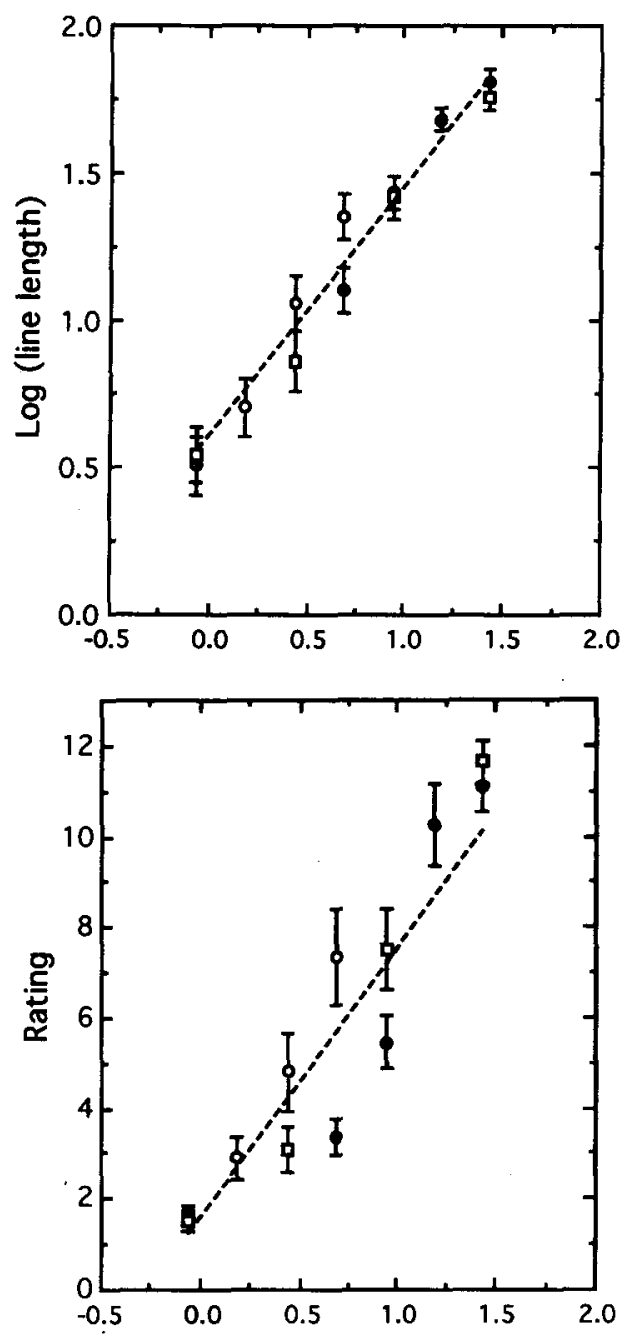

Log (sucrose concentration, \% w/v)

Figure 6. The best-fitting power function to the combined data from Experiment 1 (linear on log-log coordinates, top panel) and the best-fitting $\log$ function to the combined data from Experiment 2 (linear on linear-log coordinates, bottom panel). 
Table 1

The Parameters A and B ( $y$-Intercept and Slope) and the Chi-Square and $q$ Values of the Best-Fitting Power Functions to the Linearized Data From Experiment 1 and of the Best-Fitting Log Functions to the Linearized Data from Experiment 2

\begin{tabular}{lcccc}
\hline & A & B & Chi-Square & $q$ \\
\hline & Experiment & 1 (power function) & \\
All data & 0.615 & 0.846 & 0.735 & 0.999 \\
Weaker range & 0.540 & 1.167 & 0.025 & 0.988 \\
Stronger range & 0.606 & 0.864 & 0.233 & 0.890 \\
Extended range & 0.583 & 0.828 & 0.078 & 0.961 \\
& & & & \\
All data & Experiment 2 (log function) & & \\
Weaker range & 1.646 & 5.910 & 8.147 & 0.614 \\
Stronger range & 1.971 & 6.779 & 0.123 & 0.940 \\
Extended range & -4.031 & 10.692 & 0.486 & 0.784 \\
\hline
\end{tabular}

Note-Best-fitting functions were found for the combined data as well as for data obtained in each concentration range separately.

parameters, the chi-square value, and the probability, $q$, that the fit would have a chi-square value this large or larger (Press, Flannery, Teukolsky, \& Vetterling, 1986). The best-fitting functions to the combined data from each experiment are shown in Figure 6. Data from Experiment 1 are in the top panel, and data from Experiment 2 are in the bottom panel. About each datum is shown \pm 1 $S E$. The concordance between the parameters of the functions fitted to the combined data and the parameters of the functions fitted to individual ranges can be seen in Table 1. The parameters A and B ( $y$-intercept and slope) and the chi-square and $q$ values are given. It is evident from the variation among the parameters tabulated in Table 1 that the influence of context is less severe in Experiment 1 than in Experiment 2. This conclusion is strengthened by an examination of Figure 6, which demonstrates that in Experiment 1 there is no overlap between the standard errors of one pair of the five pairs of judgments for concentrations presented in two ranges, compared with Experiment 2, where there is no overlap between the standard errors for three of these pairs.

None of the data in this study show evidence of a stimulus-spacing bias, common in other sense modalities. This finding agrees with that of McBride (1983b), who used the rating scale of Experiment 2 to scale the intensity of sour, salty, bitter, and sweet taste stimuli. He attributed this outcome, in part, to the presentation of few concentrations per session. In McBride's experiment, different spacings were used with a single stimulus range.

If we assume that a comparison between subjective impressions originating in different senses is feasible, then an advantage for a match to the impression of line length over a match to the subjective impression of numbers is that the experience of the line arises from concurrent sensory input, and does not have to be generated mentally as numbers do in magnitude estimation. In other words, the experience of the line length and the experience of sweetness (or other sensory quality) occurs simultaneously, and each experience is linked to externally gener- ated and verifiable events. Furthermore, this method of registering sensory magnitudes might be expected to result in increased precision over magnitude estimation if subjects have a tendency to generate numbers using integer values, or preferred step sizes, for example numbers that are multiples of 5 or 10 (see, e.g., O'Mahoney \& Heintz, 1981).

With respect to context effects, the present procedure is clearly an improvement over the category-rating method, at least for the inexperienced tasters who participated in this study. A number of studies cited in the introduction suggest that AME is less context-dependent than magnitude estimation with a modulus, and the use of line-length matching was expected, on theoretical grounds, to provide a further improvement. However, the sweetness judgments recorded here were not less influenced by stimulus context than the AME loudness data of Gescheider and Hughson (1991). It is possible that the residual effects observed in both studies represent, at least in part, the effects of stimulus context upon perception rather than upon judgment, and it may be that any change in relative gain (Schneider \& Parker, 1990) or adaptation level (Marks, 1992) is of a different order in the auditory and gustatory systems. In any case, the stimulus subsets in the two studies may not have occupied comparable parts of the dynamic range of each system. It would be profitable to undertake an intentional comparison between the two approaches in the same sense modality.

\section{REFERENCES}

Birnbaum, M. H. (1982). Controversies in psychological measurement. In B. Wegener (Ed.), Social attitudes and psychophysical measurement (pp. 401-405). Hillsdale, NJ: Erlbaum.

BoGARTZ, R. S. (1979). Line ratio judgments yield subjective lengths proportional to physical lengths: Reanalysis of Engen's data. Perception \& Psychophysics, 26, 247-249.

BRYSBAERT, M. (1991). Algorithms for randomness in the behavioral sciences: A tutorial. Behavior Research Methods, Instruments, \& Computers, 23, 45-60.

Collins, A. A., \& Gescheider, G. A. (1989). The measurement of loudness in children and adults by absolute magnitude estimation and cross-modality matching. Joumal of the Acoustical Society of America, 85, 2012-2021.

Foley, H. J., Cross, D. V., \& O’Reilly, J. A. (1990). Pervasiveness and magnitude of context effects: Evidence for the relativity of absolute magnitude estimation. Perception \& Psychophysics, 48, 551-558. Gescheider, G. A. (1988). Psychophysical scaling. Annual Review of Psychology, 39, 169-200.

Gescheider, G. A., \& Hughson, B. A. (1991). Stimulus context and absolute magnitude estimation: A study of individual differences. Perception \& Psychophysics, 50, 45-57.

Hellman, R. P., \& Meiselman, C. H. (1990). Loudness relations for individuals and groups in normal and impaired hearing. Journal of the Acoustical Society of America, 88, 2596-2606.

KraNTZ, D. H. (1972). A theory of magnitude estimation and crossmodality matching. Joumal of Mathematical Psychology, 9, 168-199.

LAMING, D. (1984). The relativity of 'absolute' judgements. British Journal of Mathematical \& Statistical Psychology, 37, 152-183.

LAWLESS, H. T., \& MALONE, G. J. (1986). A comparison of rating scales: Sensitivity, replicates and relative measurement. Journal of Sensory Studies, 1, 155-174.

LEVINE, M. (1974). Geometric interpretations of some psychophysical results. In D. H. Krantz, R. C. Atkinson, R. D. Luce, \& P. Suppes (Eds.), Contemporary developments in mathematical psychology: 
Vol. 2. Measurement, psychophysics, and neural information processing (pp. 200-235). San Francisco: W. H. Freeman.

LOCKHEAD, G. R. (1992). Psychophysical scaling: Judgments of attributes or objects? Brain \& Behavioral Sciences, 15, 543-601.

Marks, L. E. (1974). On scales of sensation: Prolegomena to any future psychophysics that will come forth as science. Perception \& Psychophysics, 16, 358-376.

Marks, L. E. (1975). On coloured-hearing synesthesia: Cross-modal translations of sensory dimensions. Psychological Bulletin, 82, 303-331.

MARKS, L. E. (1978). The unity of the senses: Interrelations among the modalities. New York: Academic Press.

MARKs, L. E. (1987). On cross-modal similarity: Auditory-visual interactions in speeded discrimination. Joumal of Experimental Psychology: Human Perception \& Performance, 13, 384-394.

MarKs, L. E. (1992). The contingency of loudness perception: Context modifies equal-loudness relations. Psychological Science, 3, 285-291.

MARKS, L. E. (1993). Contextual processing of multidimensional and unidimensional auditory stimuli. Journal of Experimental Psychology: Human Perception \& Performance, 19, 227-249.

Marks, L. E., Stevens, J. C., Bartoshuk, L. M., Gent, J. F., Rifkin, B., \& STONE, V. K. (1988). Magnitude matching: The measurement of taste and smell. Chemical Senses, 13, 63-67.

MCBridE, R. L. (1983a). Category scales of sweetness are consistent with sweetness-matching data. Perception \& Psychophysics, 34, 175-179.

MCBRIDE, R. L. (1983b). A JND-scale/category-scale convergence in taste. Perception \& Psychophysics, 34, 77-83.

McBRIDE, R. L. (1986). Sweetness of binary mixtures of sucrose, fructose, and glucose. Journal of Experimental Psychology: Human Perception \& Performance, 12, 584-591.

MCBridE, R. L. (1987a). Psychophysics as measurement science, sensory science, food science. Chemistry \& Industry, 1, 25-30.

MCBride, R. L. (1987b). Taste psychophysics and the Beidler equation. Chemical Senses, 12, 323-332.

Melara, R. D., \& O'Brien, T. P. (1987). Interaction between synesthetically corresponding dimensions. Journal of Experimental Psychology: General, 116, 323-336.

Mellers, B. A. (1983a). Evidence against "absolute" scaling. Perception \& Psychophysics, 33, 523-526.

Mellers, B. A. (1983b). Reply to Zwislocki's views on "absolute" scaling. Perception \& Psychophysics, 34, 405-408.

MudD, S. A. (1963). Spatial stereotypes of four dimensions of pure tone. Journal of Experimental Psychology, 66, 347-352.

O'MAHONEY, M., \& HeINTZ, C. (1981). Direct magnitude estimation of salt taste intensity with continuous correction for salivary adaptation. Chemical Senses, 6, 101-112.

Parducci, A. (1974). Contextual effects: A range-frequency analysis. In E. C. Carterette \& M. P. Friedman (Eds.), Handbook of perception: Vol.2. Psychophysical judgment and measurement (pp. 127-143). New York: Academic Press.
Poulton, E. C. (1979). Models for biases in judging sensory magnitude. Psychological Bulletin, 86, 777-803.

Poulton, C. (1987). Bias and range effects in sensory judgments. Chemistry \& Industry, 1, 18-22.

Press, W. H., Flannery, B. R., Teukolsky, S. A., \& Vetterling, W. T. (1986). Numerical recipes. Cambridge: Cambridge University Press.

Rankin, K. M., \& MarKs, L. E. (1991). Differential context effects in taste perception. Chemical Senses, 16, 617-629.

Riskey, D. R., Parducci, A., \& Beauchamp, G. K. (1979). Effects of context in judgments of sweetness and pleasantness. Perception \& Psychophysics, 26, 171-176.

SCHNEIDER, B., \& PARKER, S. (1990). Does stimulus context affect loudness or only loudness judgments? Perception \& Psychophysics, 48, 409-418.

Stevens, S. S. (1975). Psychophysics: Introduction to its perceptual, neural and social prospects. New York: Wiley.

Teghtsoonian, M. (1965). The judgment of size. American Journal of Psychology, 78, 392-402.

TeGhtsoonian, R. (1973). Range effects in psychological scaling and a revision of Stevens' law. American Journal of Psychology, 86, 3-27.

VERRILLO, R. T. (1981). Absolute estimation of line length in three age groups. Journal of Gerontology, 36, 625-627.

WARD, L. M. (1987). Remembrance of sounds past: Memory and psychophysical scaling. Journal of Experimental Psychology: Human Perception \& Performance, 13, 216-227.

ZWISLOCKI, J. J. (1983a). Absolute and other scales: Question of validity. Perception \& Psychophysics, 33, 593-594.

ZwISLOCKI, J. J. (1983b). Group and individual relations between sensation magnitude and their numerical estimates. Perception \& Psychophysics, 33, 460-468.

ZWISLOCKI, J. J. (1991). Natural measurement. In S. J. Bolanowski, Jr., \& G. A. Gescheider (Eds.), Ratio scaling of psychological magnitude: A tribute to the memory of S. S. Stevens (pp. 18-26). Hillsdale, NJ: Erlbaum.

ZwISLOCKI, J. J., \& Goodman, D. A. (1980). Absolute scaling of sensory magnitudes: A validation. Perception \& Psychophysics, 28, 28-38.

\section{NOTES}

1. The view adopted by the present author, that in magnitude estimation subjects engage in ratio-based computation, is similar to that expre sed formally by Krantz (1972). For an alternative interpretation, see Levine (1974).

2. With respect to the labeling of the stimulus-spacing bias, I have followed Poulton (1987). He identified the stimulus-spacing bias and the stimulus-frequency bias as two distinct equal-frequency biases. The latter occurs when unequal numbers of stimuli are presented, and the observer responds as if all the stimuli were equally frequent.

(Manuscript received May 29, 1992; revision accepted for publication February 23, 1993.) 